
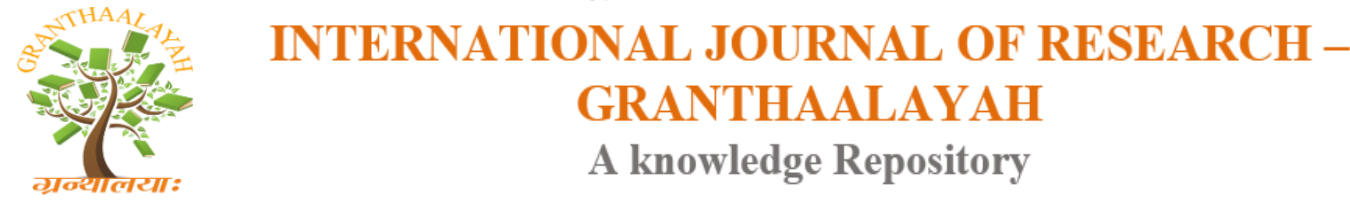

RAST - 17

\title{
CONVEXITY AND CONCAVITY OF MEANS
}

\author{
K. M. Nagaraja ${ }^{1}$, Vimala.T ${ }^{2}$ \\ ${ }^{1}$ Associate Professor, Department of Mathematics, J.S.S. Academy of Technical Education, \\ Bangalore, India \\ ${ }^{2}$ Assistant Professor, Department of Basic sciences, Jain University, India \\ DOI: https://doi.org/10.29121/granthaalayah.v5.i4RAST.2017.3309
}

\begin{abstract}
In this paper, convexity and concavity among Greek means are discussed and the results are interpreted with Vander monde's determinant.

Keywords: Convexity; Greek Means; Vander Monde's Determinant; Functions.

Cite This Article: K. M. Nagaraja, and Vimala.T. (2017). "CONVEXITY AND CONCAVITY OF MEANS." International Journal of Research - Granthaalayah, 5(4) RAST, 92-97. https://doi.org/10.29121/granthaalayah.v5.i4RAST.2017.3309.
\end{abstract}

\section{Introduction}

The well -known means are presented by Pappus of Alexandria in his books in fourth century A. D., which is the main contribution of the ancient Greeks. In Pythagorean School on the basis of proportion, ten Greek means are defined, out of which six means are named and four means are un-named. The popular named means are Arithmetic mean, Geometric mean, Harmonic mean and Contra harmonic mean. The un-named Greek means are $F_{7}(a, b), F_{8}(a, b), F_{9}(a, b)$ and $F_{10}(a, b)$ are given below; [13] here we consider the means needed for to develop this paper.

$$
\begin{gathered}
G(a, b)=\frac{a-m}{m-b}=\frac{a}{m}=\frac{m}{b}=\sqrt{a b} \\
H(a, b)=\frac{a-m}{m-b}=\frac{a}{b}=\frac{2 a b}{a+b} \\
F_{7}(a, b)=\frac{a-m}{m-b}=\frac{b}{a}=\frac{a^{2}-a b+b^{2}}{a} \\
F_{9}(a, b)=\frac{a-b}{m-b}=\frac{a}{b}=\frac{b(2 a-b)}{a}
\end{gathered}
$$

Results on convexity and concavity of one function with respect to another function were in detail discussed in [1] and also some convexity and concavity results on various important means and their applications to mean inequalities were found in $[9,11,12,14]$. 
2. Definitions and Lemmas

In this section, we shall recall some definitions and lemmas which is necessary to develop this paper.

Definition 2.a. A mean is a function $M: R_{+}^{2} \rightarrow R_{+}$, which has the property $a \wedge b \leq M(a, b) \leq a \vee b, \forall a, b>0$, where $a \wedge b=\min (a, b)$ and $a \vee b=\max (a, b)$.

Definition 2.b. Vander Monde's determinant Let $\Psi$ be a continuous function on an interval $I \subseteq R, a=\left(a_{0}, a_{1}, a_{2} \ldots \ldots \ldots a_{n}\right)$ and $a_{i} \in I, a_{i} \neq a_{j}$ for $i \neq j \quad$ see [8]. Setting

$$
V(a, \Psi)=\left|\begin{array}{cccccc}
1 & a_{0} & a_{0}^{2} & \ldots & a_{0}^{n-1} & \Psi\left(a_{0}\right) \\
1 & a_{1} & a_{1}^{2} & \ldots & a_{1}^{n-1} & \Psi\left(a_{1}\right) \\
\cdots & & \ldots & \ldots & \ldots & \ldots \\
1 & a_{n} & a_{n}^{2} & \cdots & a_{n}^{n-1} & \Psi\left(a_{n}\right)
\end{array}\right|
$$

Let $\Psi(x)=x^{n+r} \ln ^{k} x$ in 2.1, we have

$$
V(a, \Psi)=\left|\begin{array}{cccccc}
1 & a_{0} & a_{0}^{2} & \ldots & a_{0}^{n-1} & a_{0}^{n+r} \ln ^{k} a_{0} \\
1 & a_{1} & a_{1}^{2} & \ldots & a_{1}^{n-1} & a_{1}^{n+r} \ln ^{k} a_{1} \\
\ldots & \ldots & \ldots & & \ldots & \ldots \\
1 & a_{n} & a_{n}^{2} & \ldots & a_{n}^{n-1} & a_{n}^{n+r} \ln ^{k} a_{n}
\end{array}\right|
$$

Note that when $r=0$ and $k=0$ is the determinant of Vander Monde's matrix of $(n+1)^{t h}$ order. The following lemma (2.1) and (2.2) are particular cases of determinant (2.1) or (2.2)

Lemma 2.1. For $\Psi(x)=x^{2}$ and $a=\left(a_{0}, a_{1}, a_{2}\right)$ is the determinant of Vander Monde's matrix of the $3^{\text {rd }}$ order takes the form:

$$
V(a ; r=0, k=0)=\left|\begin{array}{lll}
1 & a_{0} & a_{0}^{2} \\
1 & a_{1} & a_{1}^{2} \\
1 & a_{2} & a_{2}^{2}
\end{array}\right|
$$

which is equivalently

$$
V(a ; r=0, k=0)=\left(a_{1}-a_{0}\right)\left(a_{2}-a_{0}\right)\left(a_{2}-a_{1}\right)
$$

Lemma 2.2. For $\Psi(x)=x^{1 / 2}=\sqrt{x}$ and $a=\left(a_{0}, a_{1}, a_{2}\right)$ is the determinant of Vander Monde's matrix of $3^{\text {rd }}$ order takes the form:

which is equivalently

$$
V(a ; r=0, k=0)=\left|\begin{array}{ccc}
1 & a_{0} & \sqrt{a_{0}} \\
1 & a_{1} & \sqrt{a_{1}} \\
1 & a_{2} & \sqrt{a_{2}}
\end{array}\right|
$$

$$
V(a ; r=-3 / 2, k=0)=\left(\sqrt{a_{1}}-\sqrt{a_{0}}\right)\left(\sqrt{a_{2}}-\sqrt{a_{0}}\right)\left(\sqrt{a_{2}}-\sqrt{a_{1}}\right)
$$

Lemma 2.3. [1] Let $h(x)$ and $k(x)$ are two functions, then $k(x)$ is said to be convex with respect to $h(x)$ for $a \leq b \leq c$ if and only if 


$$
\left|\begin{array}{lll}
1 & h(a) & k(a) \\
1 & h(b) & k(b) \\
1 & h(c) & k(c)
\end{array}\right| \geq 0 \simeq\left|\begin{array}{ccc}
1 & h(a) & k(a) \\
0 & h(b)-h(a) & k(b)-k(a) \\
0 & h(c)-h(a) & k(c)-k(a)
\end{array}\right| \geq 0
$$

which is equivalently

$$
[h(b)-h(a)][k(c)-k(a)]-[h(c)-h(a)][k(b)-k(a)] \geq 0 .
$$

Setting $a=x$ and $b=1$ in eqs (1.a) to (1.d), The popular named means Geometric mean, Harmonic mean. The un-named means $F_{7}(a, b)$ and $F_{9}(a, b)$ takes the following form:

(i) $G(x, 1)=\sqrt{x}$

ii) $H(x, 1)=\frac{2 x}{x+1}$

iii) $F_{7}(x, 1)=\frac{x^{2}-x+1}{x}$

iv) $F_{9}(x, 1)=\frac{(2 x-1)}{x}$

\section{Main Results}

In this section, the necessary and sufficient conditions for Convexity and Concavity of different means are discussed and the results are expressed in terms of Vander Monde's determinants.

Theorem 3.1. The Harmonic mean is concave (convex) with respect to $F_{7}(a, b)$ if and only if $V(a ; r=0, k=0) \leq(\geq) 0$.

Proof: Consider the Harmonic mean and $F_{7}(a, b)$ in the form;

$$
\begin{gathered}
H(x, 1)=\frac{2 x}{x+1} \text { and } F_{7}(x, 1)=\frac{x^{2}-x+1}{x} \\
\text { Let } \quad h(x)=\frac{2 x}{x+1} \\
\text { and } k(x)=\frac{x^{2}-x+1}{x}, \text { by lemma }(2.3) \text { we have } \\
\left|\begin{array}{ccc}
1 & h(a) & k(a) \\
0 & h(b)-h(a) & k(b)-k(a) \\
0 & h(c)-h(a) & k(c)-k(a)
\end{array}\right|=\left|\begin{array}{ccc}
1 & \frac{2 a}{a+1} & \frac{a^{2}-a+1}{a} \\
0 & \frac{2(b-a)}{(a+1)(b+1)} & \frac{b^{2}-b+1}{b}-\frac{a^{2}-a+1}{a} \\
0 & \frac{2(c-a)}{(c+1)(a+1)} & \frac{c^{2}-c+1}{c}-\frac{a^{2}-a+1}{a}
\end{array}\right|
\end{gathered}
$$

On Simplifying the determinant leads to

$\frac{2(b-a)(c-a)(c-b)(a b c+1)}{2 a b c(a+1)(b+1)(c+1)} \geq 0$.

By lemma (2.1) we have

$$
\frac{2(b-a)(c-a)(c-b)(a b c+1)}{2 a b c(a+1)(b+1)(c+1)}=\frac{2 V(a ; r=0, k=0)(a b c+1)}{2 a b c(a+1)(b+1)(c+1)} \geq 0
$$

By lemma (2.3), $0<a<b<c$ implies that $2 a b c(a+1)(b+1)(c+1) \geq 0$

Similarly by considering $h(x)=\frac{x^{2}-x+1}{x}$ and $k(x)=\frac{2 x}{x+1}, \quad$ by lemma (2.3) we have

$$
\frac{2(b-a)(c-a)(b-c)(a b c+1)}{2 a b c(a+1)(b+1)(c+1)}=\frac{2 V(a ; r=0, k=0)(a b c+1)}{2 a b c(a+1)(b+1)(c+1)} \leq 0
$$

By combining eqs (3.1) and (3.2) the proof of theorem (3.1) completes. 
Theorem 3.2. The Harmonic mean is concave (convex) with respect to $F_{9}(a, b)$ if and only if $V(a ; r=0, k=0) \leq(\geq) 0$.

Proof: Consider the Harmonic mean and $F_{9}(a, b)$ in the form;

$$
H(x, 1)=\frac{2 x}{x+1} \text { and } F_{9}(x, 1)=\frac{2 x-1}{x}
$$

Let $\quad h(x)=\frac{2 x}{x+1}$ and $k(x)=\frac{2 x-1}{x}$, by lemma (2.3) we have

$$
\left|\begin{array}{ccc}
1 & h(a) & k(a) \\
0 & h(b)-h(a) & k(b)-k(a) \\
0 & h(c)-h(a) & k(c)-k(a)
\end{array}\right|=\left|\begin{array}{ccc}
1 & \frac{2 a}{a+1} & \frac{2 a-1}{a} \\
0 & \frac{2(b-a)}{(a+1)(b+1)} & \frac{2 b-1}{b}-\frac{2 a-1}{a} \\
0 & \frac{2(c-a)}{(a+1)(b+1)} & \frac{2 b-1}{b}-\frac{2 a-1}{a}
\end{array}\right|
$$

On Simplifying the determinant leads to

$$
\frac{2(b-a)(c-a)(c-b)}{a b c(a+1)(b+1)(c+1)}=\frac{\frac{2(b-a)(c-a)(c-b)}{a b c(a+1)(b+1)(c+1)} \geq 0}{a b c(a+1)(b+1)(c+1)} \geq 0
$$

Assume that $0<a<b<c$ implies that $a b c(a+1)(b+1)(c+1) \geq 0$

Similarly by considering $h(x)=\frac{2 x-1}{x}$ and $k(x)=\frac{2 x}{x+1}$, by lemma (2.3) we have $\frac{2(b-a)(c-a)(b-c)}{a b c(a+1)(b+1)(c+1)}=\frac{2 V(a ; r=0, k=0)}{a b c(a+1)(b+1)(c+1)} \leq 0$

By combining eqs (3.3) and (3.4) the proof of theorem (3.2) completes.

Theorem 3.3. The Geometric mean is concave (convex) with respect to $F_{7}(a, b)$ if and only if $V(a ; r=-3 / 2, k=0) \leq(\geq) 0$.

Proof: Consider the Geometric mean and $F_{7}(a, b)$ in the form;

$$
G(x, 1)=\sqrt{x} \text { and } F_{7}(x, 1)=\frac{x^{2}-x+1}{x}
$$

Let $h(x)=\sqrt{x}$ and $k(x)=\frac{x^{2}-x+1}{x}$, by lemma (2.3) we have

$$
\left|\begin{array}{ccc}
1 & h(a) & k(a) \\
0 & h(b)-h(a) & k(b)-k(a) \\
0 & h(c)-h(a) & k(c)-k(a)
\end{array}\right|=\left|\begin{array}{ccc}
1 & \sqrt{a} & \frac{a^{2}-a+1}{a} \\
0 & \sqrt{b}-\sqrt{a} & \frac{b^{2}-b+1}{b}-\frac{a^{2}-a+1}{a} \\
0 & \sqrt{c}-\sqrt{a} & \frac{c^{2}-c+1}{c}-\frac{a^{2}-a+1}{a}
\end{array}\right|
$$

On Simplifying the determinant leads to

$$
\frac{(\sqrt{b}-\sqrt{a})(\sqrt{c}-\sqrt{a})(\sqrt{c}-\sqrt{b})}{a b c}(a b c+\sqrt{a c}+\sqrt{a b}+\sqrt{b c}) \geq 0
$$

Then by lemma (2.2)

$$
\begin{aligned}
& \frac{(\sqrt{b}-\sqrt{a})(\sqrt{c}-\sqrt{a})(\sqrt{c}-\sqrt{b})}{a b c}(a b c+\sqrt{a c}+\sqrt{a b}+\sqrt{b c}) \\
= & \frac{v(a ; r=-3 / 2, k=0)}{a b c}(a b c+\sqrt{a c}+\sqrt{a b}+\sqrt{b c}) \geq 0
\end{aligned}
$$

Similarly by considering $h(x)=\frac{x^{2}-x+1}{x}$ and $k(x)=\sqrt{x}$, by lemma (2.3) we have $\frac{(\sqrt{b}-\sqrt{a})(\sqrt{c}-\sqrt{a})(\sqrt{c}-\sqrt{b})}{a b c}(a b c+\sqrt{a c}+\sqrt{a b}+\sqrt{b c}) \leq 0$

By combining eqs (3.5) and (3.6) the proof of theorem (3.3) completes. 
Theorem 3.4. The Geometric mean is concave (convex) with respect to $F_{9}(a, b)$ if and only if $V(a ; r=-3 / 2, k=0) \leq(\geq) 0$.

Proof: Consider the Geometric mean and $F_{9}(a, b)$ in the form;

$$
G(x, 1)=\sqrt{x} \text { and } F_{9}(x, 1)=\frac{2 x-1}{x}
$$

Let $h(x)=\sqrt{x}$ and $k(x)=\frac{2 x-1}{x}$, by lemma (2.3) we have

$$
\left|\begin{array}{ccc}
1 & h(a) & k(a) \\
0 & h(b)-h(a) & k(b)-h(a) \\
0 & h(c)-h(a) & k(c)-k(a)
\end{array}\right|=\left|\begin{array}{ccc}
1 & \sqrt{a} & \frac{2 a-1}{a} \\
0 & \sqrt{b}-\sqrt{a} & \frac{2 b-1}{b}-\frac{2 a-1}{a} \\
0 & \sqrt{c}-\sqrt{a} & \frac{2 c-1}{c}-\frac{2 a-1}{a}
\end{array}\right|
$$

On Simplifying the determinant leads to

$\frac{(\sqrt{b}-\sqrt{a})(\sqrt{c-}-\sqrt{b})(\sqrt{c}-\sqrt{b})}{a b c}(\sqrt{a c}+\sqrt{a b}+\sqrt{b c}) \leq 0$

By lemma (2.2) we have

$$
\begin{aligned}
& \frac{(\sqrt{b}-\sqrt{a})(\sqrt{c}-\sqrt{b})(\sqrt{b}-\sqrt{c})}{a b c}(\sqrt{a c}+\sqrt{a b}+\sqrt{b c}) \\
= & \frac{(\sqrt{b}-\sqrt{a})(\sqrt{c}-\sqrt{b})(\sqrt{b}-\sqrt{c})}{a b c}(\sqrt{a c}+\sqrt{a b}+\sqrt{b c}) \leq 0
\end{aligned}
$$

Similarly by considering $h(x)=\frac{x^{2}-x+1}{x}$ and $k(x)=\sqrt{x}$, by lemma (2.3) we have

$$
\frac{(\sqrt{b}-\sqrt{a})(\sqrt{c-}-\sqrt{b})(\sqrt{b}-\sqrt{c})}{a b c}(\sqrt{a c}+\sqrt{a b}+\sqrt{b c}) \geq 0
$$

By combining eqs (3.7) and (3.8) the proof of theorem (3.4) completes.

Theorem 3.5: The unnamed mean $F_{7}(a, b)$ is concave (convex) with respect to $F_{9}(a, b)$ if and only if $V(a ; r=0, k=0) \leq(\geq) 0$

Proof: Consider $F_{7}(a, b)$ and $F_{9}(a, b)$ in the form;

$$
F_{7}(x, 1)=\frac{x^{2}-x+1}{x} \text { and } F_{9}(a, b)=\frac{2 x-1}{x}
$$

Let $h(x)=\frac{x^{2}-x+1}{x}$ and $k(x)=\frac{2 x-1}{x}$

By lemma 2.3, we have

$$
\left|\begin{array}{ccc}
1 & h(a) & k(a) \\
0 & h(b)-h(a) & k(b)-h(a) \\
0 & h(c)-h(a) & k(c)-k(a)
\end{array}\right|=\left|\begin{array}{ccc}
1 & \frac{a^{2}-a+1}{a} & \frac{2 a-1}{a} \\
0 & \frac{b^{2}-b+1}{b}-\frac{a^{2}-a+1}{a} & \frac{2 b-1}{b}-\frac{2 a-1}{a} \\
0 & \frac{c^{2}-c+1}{c}-\frac{a^{2}-a+1}{a} & \frac{2 c-1}{c}-\frac{2 a-1}{a}
\end{array}\right|
$$

On simplifying the determinant leads to $\frac{(b-a)(c-a)(b-c)}{a b c} \leq 0$

By lemma 2.1, we have

$$
\frac{(b-a)(c-a)(b-c)}{a b c}=\frac{V(a ; r=0, k=0)}{a b c} \leq 0
$$

Similarly by considering $h(x)=\frac{2 x-1}{x}$ and $k(x)=\frac{x^{2}-x+1}{x}$

By lemma 2.3, we have $\frac{(b-a)(c-a)(b-c)}{a b c} \geq 0$

By combining eqs (3.9) and (3.10) the proof of theorem (3.5) completes. 


\section{References}

[1] P. S. Bullen, Hand book of Means and Their Inequalities, Kluwer Academic Publishers, Dordrecht, 2003.

[2] G. H. Hardy, J. E. Littlewood and G. Polya, Inequalities, Cambridge University Press, Cambridge, 1959.

[3] V.Lokesha and K. M. Nagaraja, Relation between series and important means, Advances in Theoretical and Applied Mathematics,2(1) (2007), 31-36.

[4] V. Lokesha, K. M. Nagaraja and Yilmaz Simsek, New Inequalities on the homogeneous functionsJ. Indone. Math. Soc., 15(1) (2009), 49-59.

[5] V. Lokesha, Zhi-Hua Zhang and K. M. Nagaraja, Gnan mean for two variables, Far East Journal of Applied Mathematics, 31(2) (2008), 263-272.

[6] K. M. Nagaraja, Zhi-Hua Zhang, V. Lokesha and Zhen-Gang Xiao, Generalization of the weighted Heron mean in n variables, The Aligarh Bulletin of Mathematics, 26(1) (2007), 21-27.

[7] K. M. Nagaraja, V. Lokesha and S.Padmanabhan, A simple proof on strengthening and extension of inequalities, Adv. Stud. Contemp. Math, 17(1) (2008), 97-103.

[8] K. M. Nagaraja, V. Lokesha, Zhen-Gang Xiao and Zhi-Hua Zhang, The Generalized Weighted Elementary Symmetric Mean, Ultra Scientist for Physical Sciences, 22(2) (2010),651-657.

[9] K. M. Nagaraja and P.S.K.Reddy, Logarithmic convexity of Double sequences, Scientia Magna, $7(2)(2011), 78-81$.

[10] K. M. Nagaraja and P. S. K. Reddy, alpha-Centroidal mean and its dual, Proceedings of the Jangjeon Math. Soc.15 (2012), N0.2, pp. 163-170.

[11] J. Rooin and M. Hassni, Some new inequalities between important means and applications to \$Ky\$-Fan types inequalities, Math.Ineq. and Appl, 10(3) (2007), 517-527.

[12] Slavko Simic, An extension of Stolarsky means,Novi Sad J. Math.38(3) (2008), 81-89.

[13] G.Toader and S. Toader, Greek means and Arithmetic mean and Geometric mean, RGMIA Monograph, (2005), Australia.

[14] R. Webster, Convexity, Oxford University Press, New York, 1994.

[15] Zhen-Gang Xiao, Zhi-Hua Zhang, V. Lokesha and K. M. Nagaraja, A class of new three parameter generalized weighted mean, Int. J. Appl. Math. Stat. 11(7) (2007),193-202.

*Corresponding author.

E-mail address: nagkmn@gmail.com 